\title{
Three-Dimensional Nanostructure Determination Based On Scanning Electron Nanodiffraction
}

Yifei Meng ${ }^{1,2}$ and Jian-Min Zuo ${ }^{1,2}$

1. Department of Materials Science \& Engineering, University of Illinois at Urbana-Champaign, Urbana, Illinois, USA

2. Fredrick Seitz Materials Research Laboratory, University of Illinois at Urbana-Champaign, Urbana, Illinois, USA

Nanocrystalline materials in general are structurally featured by a large density of grain boundaries and large surface/volume ratio, which have attracted significant interest for their unique mechanical, chemical and electronic properties [1-3]. Transmission electron diffraction (TED) is an appropriate technique for complex nanostructure analysis because it is highly sensitive to local structure and it can be obtained using a small electron beam. Previously we have developed a TEM based scanning electron nanodiffraction (SEND) technique that uses the built-in TEM deflection coils to shift the beam [4].

Here, we report a new technique called 3D-SEND by coupling the SEND with diffraction tomography. This technique aims at determining the 3D morphologies and orientations of grains in nanocrystalline materials from a large set of diffraction patterns. A special holder design is employed for a high-angle (up to $87^{\circ}$ ) sample rotation [5]. The design employs a needle-shape specimen and a sample rotation driven by the goniometer itself. Diffraction pattern recording and beam scanning are automated using a DigitalMicrograph $^{\circledR}$ script to control the TEM deflection coils and camera readout [6]. A stack of diffraction patterns are acquired for each sample rotation step.

For each scattering spot, we reconstruct a dark-field image using the 2D SEND dataset. In order to identify 2D grain morphologies, dark-field images with similar contrasts are grouped using the normalized cross-correlation. The dark-field images in one group are further averaged. The 2D morphology is extracted from the averaged image with an intensity threshold. Meanwhile, scattering spots belong to one grain are effectively grouped into one diffraction pattern. Electron diffraction pattern indexing is achieved by a combination of diffraction peak search and peak indexing using both length and angle information. During the sample rotation, a grain can be observed at a certain range of angles. Observable 2D morphologies of the grain are used as the projections for the 3D reconstruction. The tomographic reconstruction of the grain is performed using the algebraic reconstruction technique. We apply prior conditions concerning the sample outline and the damping of scattering intensity. A smoothed isosurface is created to illustrate the grain's 3D morphology. The grain orientation is determined by considering indexing results from the rotation range.

We demonstrate the performance of 3D-SEND on a TiN thin-film nanocrystalline sample prepared by unbalanced magnetron sputtering. The FIB cut and lift-out was performed perpendicular to the growth direction. The sample was milled to a tip with a diameter of $200 \mathrm{~nm}$. The beam was set to $7 \mathrm{~nm}$ in FWHM. The scanning covered a $26 \times 26$ pixels area and each step is $11 \mathrm{~nm}$. The sample was tilted over a range of $\pm 85^{\circ}$ with a step of $5^{\circ}$. In total 23660 DPs are recorded. Figure 1 shows the reconstruction results of seven major grains and their orientations. The reconstruction results show that grains are elongated and the grain boundaries are not ideally flat. 
The spatial resolution of this technique is ultimately limited by the electron probe size under the column approximation. For a JEOL 2100 TEM, we can form a probe with a FWHM of $2.3 \mathrm{~nm}$ using a $10 \mathrm{um}$ condenser aperture in the CBD mode with $\alpha=1$. Compared with the alternative technique 3D-OMiTEM [7], 3D-SEND has a better diffraction pattern resolution, a wider sample rotation range and a much lower electron dose. Our approach also grants a combinative study with other techniques such as atomprobe tomography and in-situ deformation. In the future, 3D-SEND may potentially be improved for the five-parameter characterization of grain boundaries [8].

\section{References:}

[1] E. Abe et al, Acta Materialia, 50(2002), p. 3845-3857.

[2] D. Wang et al, ACS Nano, 3(2009), p. 907-914.

[3] C. C. Koch et al, MRS Bulletin, 24(1999), p. 54-58.

[4] K. H. Kim et al, Micron, 71(2015), p. 39-45.

[5] S. Mao et al, Acta Materialia, 82(2015), p. 328-335.

[6] J. Tao et al, Physical Review Letters, 103(2009), p. 097202.

[7] H. H. Liu et al, Science, 332(2011), p. 833-834.

[8] We thank Prof. Huang of National Tsinghua University for providing the TiN sample. We thank Dr. Mao and Prof. Dillon of University of Illinois at Urbana-Champaign for providing the design of the tomography holder. This work is supported by U.S. Department of Energy under contract DEFG02$01 E R 45923$.

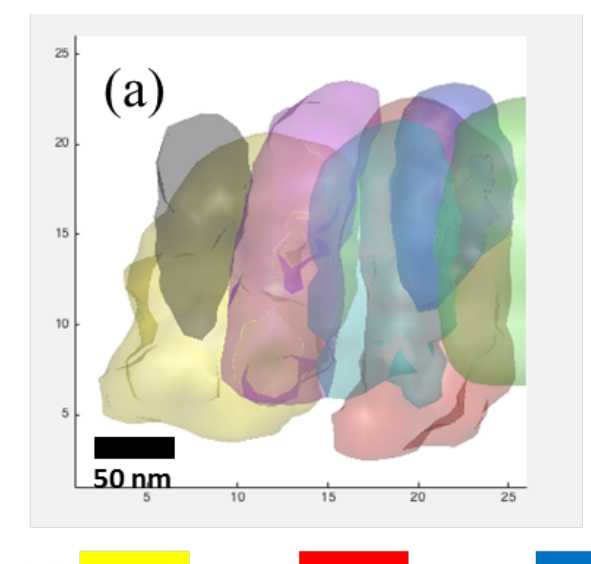

(d)
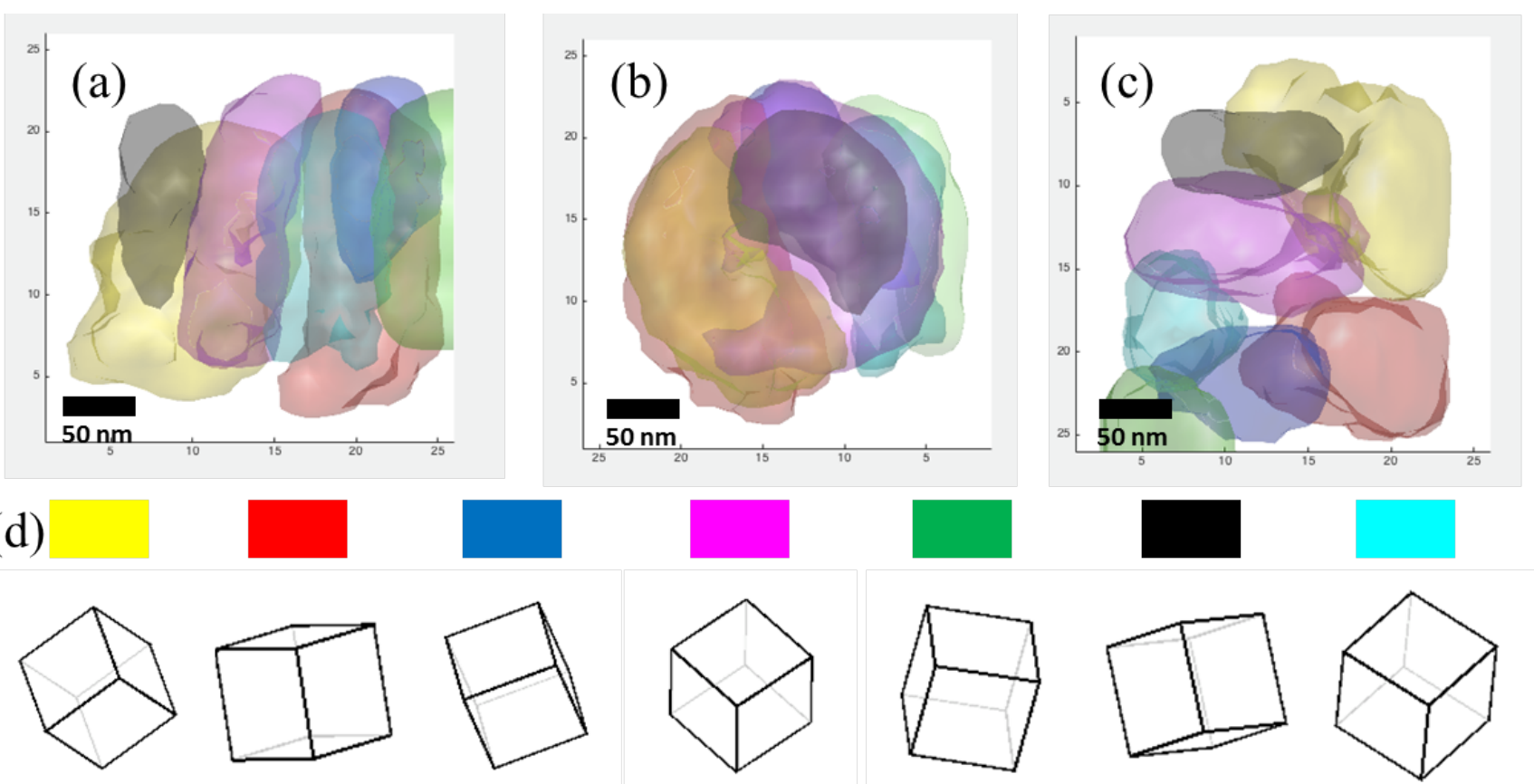

Figure 1. Side (a), front (b) and top view (c) of the 3D morphologies of reconstructed grains. Each grain is represented by one color. (d) Orientations of the seven grains. The orientation cube is labeled by the color of the grain. 